\title{
Systems pharmacogenomics identifies novel targets and clinically actionable therapeutics for medulloblastoma
}

Laura A. Genovesi 1,2, Amanda Millar', Elissa Tolson', Matthew Singleton', Emily Hassall', Marija Kojic, Caterina Brighi ${ }^{3,4}$, Emily Girard ${ }^{5}$, Clara Andradas ${ }^{6}$, Mani Kuchibhotla $^{6}$, Dharmesh D. Bhuva ${ }^{7,8}$, Raelene Endersby ${ }^{6}$, Nicholas G. Gottardo ${ }^{6}$, Anne Bernard ${ }^{9}$, Christelle Adolphe', James M. Olson ${ }^{5}$, Michael D. Taylor ${ }^{10,11}$, Melissa J. Davis ${ }^{7,8,12^{*}+}$ and Brandon J. Wainwright ${ }^{1,2^{*}+}$

\begin{abstract}
Background: Medulloblastoma (MB) is the most common malignant paediatric brain tumour and a leading cause of cancer-related mortality and morbidity. Existing treatment protocols are aggressive in nature resulting in significant neurological, intellectual and physical disabilities for the children undergoing treatment. Thus, there is an urgent need for improved, targeted therapies that minimize these harmful side effects.

Methods: We identified candidate drugs for MB using a network-based systems-pharmacogenomics approach: based on results from a functional genomics screen, we identified a network of interactions implicated in human MB growth regulation. We then integrated drugs and their known mechanisms of action, along with gene expression data from a large collection of medulloblastoma patients to identify drugs with potential to treat MB.

Results: Our analyses identified drugs targeting CDK4, CDK6 and AURKA as strong candidates for MB; all of these genes are well validated as drug targets in other tumour types. We also identified non-WNT MB as a novel indication for drugs targeting TUBB, CAD, SNRPA, SLC1A5, PTPRS, P4HB and CHEK2. Based upon these analyses, we subsequently demonstrated that one of these drugs, the new microtubule stabilizing agent, ixabepilone, blocked tumour growth in vivo in mice bearing patient-derived xenograft tumours of the Sonic Hedgehog and Group 3 subtype, providing the first demonstration of its efficacy in MB.
\end{abstract}

Conclusions: Our findings confirm that this data-driven systems pharmacogenomics strategy is a powerful approach for the discovery and validation of novel therapeutic candidates relevant to MB treatment, and along with data validating ixabepilone in PDX models of the two most aggressive subtypes of medulloblastoma, we present the network analysis framework as a resource for the field.

Keywords: Medulloblastoma, Genetic screen, Protein interaction network, Drug target, Microtubule stabilization

\footnotetext{
*Correspondence: davis.m@wehi.edu.au; b.wainwright@imb.uq.edu.au

${ }^{\dagger}$ Melissa J. Davis and Brandon J. Wainwright jointly supervised the study.

${ }^{7}$ Bioinformatics Division, The Walter and Eliza Hall Institute of Medical

Research, Parkville, Victoria 3052, Australia

${ }^{1}$ The University of Queensland Diamantina Institute, The University of

Queensland, Woolloongabba, QLD 4102, Australia

Full list of author information is available at the end of the article
}

(C) The Author(s). 2021 Open Access This article is licensed under a Creative Commons Attribution 4.0 International License, which permits use, sharing, adaptation, distribution and reproduction in any medium or format, as long as you give appropriate credit to the original author(s) and the source, provide a link to the Creative Commons licence, and indicate if changes were made. The images or other third party material in this article are included in the article's Creative Commons licence, unless indicated otherwise in a credit line to the material. If material is not included in the article's Creative Commons licence and your intended use is not permitted by statutory regulation or exceeds the permitted use, you will need to obtain permission directly from the copyright holder. To view a copy of this licence, visit http://creativecommons.org/licenses/by/4.0/ The Creative Commons Public Domain Dedication waiver (http://creativecommons.org/publicdomain/zero/1.0/) applies to the data made available in this article, unless otherwise stated in a credit line to the data. 


\section{Background}

Medulloblastoma (MB) is the most common malignant paediatric brain tumour accounting for $20-25 \%$ of all childhood brain cancers [1]. Remarkable progress in defining the molecular and cytogenetic features of MB has confirmed the biological heterogeneity of $\mathrm{MB}$, with several studies defining at least four molecular subgroups: Wingless (WNT), Sonic Hedgehog (SHH), Group 3 (Gp3) and Group 4 (Gp4) and up to 12 subtypes within the subgroups [2-7]. Current treatment options including surgical resection, craniospinal irradiation and highdose chemotherapy have improved survival rates to approximately $70-75 \%$ for children with average-risk MB [8-11]. The outcome for high-risk patients however remains poor and survivors frequently relapse and face significant long-term neurocognitive, endocrine and physical sequelae as a consequence of aggressive treatment protocols. Several studies have demonstrated that the stratification of patients on the basis of the distinct molecular features defining $\mathrm{MB}$ subgroups can have a profound impact on their clinical outcome (as reviewed in [12]), warranting the integration of subgroup-specific molecular profiles into the stratification of patients and ultimately, the designation of treatment protocols. A multicentre clinical trial investigating the stratification of patients on the basis of molecular subgroup (WNT, $\mathrm{SHH}$ or non-WNT/ non-SHH) followed by clinical risk (low, average or high-risk) (SJMB12) is currently underway. This trial will evaluate whether the addition of the SHH pathway Smoothened (SMO) antagonist, vismodegib, and new chemotherapeutic agents, gemcitabine and pemetrexed, to standard chemotherapy will improve treatment outcome for patients with $\mathrm{SHH} \mathrm{MB}$ and non$\mathrm{WNT} /$ non-SHH $\mathrm{MB}$ respectively. In parallel to this study, the identification and development of additional novel targeted therapeutic options aimed towards the specific molecular drivers of $\mathrm{MB}$ is urgently required. Ultimately, this will enable a new era in the treatment of $\mathrm{MB}$, where patients are preselected for a particular targeted therapy based on molecular phenotype, an approach that will improve clinical benefit and may lead to a reduction in the toxicity associated with existing nonprecision treatments.

Systems pharmacogenomics, an approach that integrates computational modelling with biomedical, biomolecular and genomic data, has the potential to make a substantial contribution to the development of novel therapeutics or to the repurposing of existing drugs for new diseases. Whilst pharmacogenomics sets out a framework for identifying the relationship between the genome and drug response, systems pharmacogenomics places this work in the context of systems biology. By identifying how the network of interacting genes and proteins cooperate to dictate drug response, we can use patterns in the network to target therapy. Pharmacogenomics approaches have shown benefit in other cancers $[13,14]$; however, the scarcity of actionable mutations in $\mathrm{MB}$ has historically limited the application of genomics for drug selection [15]. The development of a computational strategy for prioritizing candidate drugs represents an alternative approach to rapidly evaluate existing therapeutics against a computational model prior to moving to experimental work.

Here, we present a systems approach to the identification of novel drug targets for all non-WNT MB, using a protein-protein interaction network constructed from genes identified as promoting aggressive disease in our transposon-driven mouse model of MB and experimentally verified, literature curated protein-protein interactions (PPI) [16]. Briefly, Sleeping Beauty (SB)-induced mutagenesis in the Patched 1 (Ptch1) heterozygous mouse model resulted in accelerated MB tumorigenesis, with transposon common insertion sites (CIS) determined to identify candidate causative candidate cancer genes driving accelerated MB development [16]. The local protein network for each CIS-derived candidate cancer gene was generated from experimentally determined PPI data and these local protein networks were integrated to generate a protein interaction network comprising the CISs and their interacting proteins. Unexpectedly, the CIS-derived candidate cancer genes and associated protein network was capable of distinguishing the molecular subgroups of human $\mathrm{MB}$, indicating that the $S B$ mouse model of MB captured the genetic diversity and common pathways underpinning distinctive human MB subgroups [16]. Given the power of this integrated computational and experimental approach to predict the complex biology underlying $\mathrm{MB}$, here we have used this functionally defined PPI network to define novel therapeutic approaches for all molecular subgroups of human $\mathrm{MB}$.

We restricted this analysis to non-WNT MB since the WNT subgroup is associated with greater than 95\% long-term survival and is by some margin the least frequent subgroup. We chose to focus on over-expressed genes in human $\mathrm{MB}$, given that majority of drugs are inhibitors and block protein function. Additionally, elevated mRNA expression has been identified as a strong characteristic hallmark in the computational identification of novel anti-cancer drug targets using high-throughput data [17]. Working within the drug-repurposing paradigm, we created a drug-target network using the DrugBank database and significantly over-expressed genes identified from human $\mathrm{MB}$ expression data (Additional file 1: Fig. S1). We then identified druggable targets, defined exclusively as proteins with validated drug interactions rather than proteins with predicted drug interactions. Additionally, we focused on protein network/drug combinations 
that were in common between $\mathrm{SHH}, \mathrm{Gp} 3$ and Gp4 MB on the basis that an ideal therapeutic would target all three subgroups. Such therapeutics are likely to have the greatest clinical impact with, ultimately, a simplified clinical trial design afforded by targeting three tumour subgroups simultaneously.

Several of the targets we predicted by this approach, including Aurora kinase A (AURKA), cyclin-dependent kinase 6 (CDK6), cyclin-dependent kinase 4 (CDK4) and checkpoint kinase 2 (CHEK2), are validated targets in $\mathrm{MB}$ and currently have drugs targeting them under development as $\mathrm{MB}$ therapeutics [18-21], lending weight to the novel predictions that also arose from our analysis. This principled and data-driven systems pharmacology approach not only identified new and existing protein targets but also identified a network of therapeutics that would potentially target those proteins. Here, one such therapeutic, ixabepilone, targeting the functional hub tubulin beta chain (TUBB) defined in our analyses was tested in $\mathrm{Gp} 3$ and $\mathrm{SHH}$ patient-derived xenograft (PDX) models leading to the identification of considerable anti-tumour activity in all cases. Our experimental data in Gp3 and SHH PDX MB coupled with the success of many network leads as drug targets validates the capability of this drug-target network to identify common therapeutic strategies for multiple subgroups of $\mathrm{MB}$.

\section{Methods}

\section{Bioinformatics and statistics}

$S B$-mutagenesis CIS-derived candidate cancer genes were identified and mapped to mouse and human protein coding genes as described in our previous study [16]. Local protein interaction networks for each $S B$-mutagenesis CIS-derived candidate cancer gene were constructed to generate a protein interaction network consisting of 622 proteins and 666 edges as previously described [16]. Cytoscape 3.2.0 was employed to analyse and visualize the network [22]. Human primary MB $(n=285)$ were profiled on the Affymetrix Human Exon 1.1ST 24Array platform at the German Cancer Research Center (DKFZ, www.dkfz.de; Heidelberg, Germany, available through the Gene Expression Ombibus: GSE37382). Sample annotation was collected from the GSE37382 series matrix file. Expression data were $\log _{2}$ transformed and $z$ score normalized using R. Differential expression was calculated using the LIMMA package in Bioconductor. A threshold of 0.05 was applied to the FDR corrected $P$ value to identify significantly differentially expressed genes relative to normal foetal and adult cerebellum. Normal foetal and adult cerebellum samples (GSE167447) were processed in the same way. All data were then mean centred and scaled to ensure comparable distributions prior to differential expression analysis.
Probes corresponding to the proteins residing in our interaction network were selected based on the probe annotation file for GPL11532, and expression data were extracted from the GSE37382 Series Matrix file. No additional probe selection was applied, and if more than one probe corresponded to a listed gene, all probes for that gene were selected. Expression data were imported into our protein interaction network to identify over-expressed genes present in the network. Drugs and their molecular targets were obtained from DrugBank version 4.3 [23] and integrated with the protein interaction network to create a drug-target protein interaction network consisting of 1274 nodes and 1435 edges. Briefly, human protein-protein interaction data was collected from International Molecular Exchange (IMEX) Consortium partner databases [24]. Hits from the mutagenesis screen were mapped to human orthologs, and protein interactions involving those orthologs were retrieved and used to construct the base protein interaction network. We then retrieved drugs known to target proteins in this network from Drug Bank and added drug-protein interactions to the base protein interaction network. This protein-protein-drug network was used for all subsequent analyses.

For subgroup-specific analysis, a refined protein interaction network was created including only the significantly over-expressed proteins $(F D R P<0.05)$ and their direct protein-protein interactors. The number of patient samples, significantly deregulated proteins, putative drug targets, putative drugs and the nodes and edges contained in each of the subgroup-specific networks is included in Additional file 2: Tables S1 and Additional file 3: Table S2. Drugs and their molecular targets were mapped to proteins residing in this over-expressed network, creating drug-target networks specific to each $\mathrm{MB}$ subgroup, and to identify significantly over-expressed druggable proteins specific to each individual subgroup of MB. Differential expression data was integrated with the base network, with the results (fold change and $\mathrm{p}$ value) of all four comparisons added to protein nodes as node attributes. For each comparison, we then filtered nodes to select those that were significantly upregulated and then generated a sub-network that included the direct neighbours of these upregulated nodes (that is, we selected additional nodes one step out from the upregulated protein). This expansion step pulled in both neighbouring proteins, and any drugs targeting the upregulated proteins, from the base network described above. Expression levels of tubulin beta class 1 (TUBB) in MB PDXs were obtained from microarray analysis results from the R2 Genomics Analysis and Visualization Platform (http://r2.amc.nl) using the Mixed Paediatric PDX (public) dataset. TUBB probesets in each database with the highest average signals were selected for analysis. 


\section{Human cohorts}

Human gene expression data for medulloblastoma samples (GSE37382) and normal foetal and adult cerebellum samples (GSE167447) were downloaded from the Gene expression Omnibus and processed as described above. All data are publicly available: see the "Availability of data and materials" section for details.

\section{Mice}

Six to ten-week-old NOD.Cg-Prkdc $c^{\text {scid }} I l 2 r g^{\text {tm } 1 \text { Wjl }} / S z J$ (NSG) mice were originally purchased from The Jackson Laboratory (Bar Harbor, ME) and maintained as a colony at the Translational Research Institute, University of Queensland. Female athymic nude mice (Hsd:Athymic Nude-Foxn $\left.1^{n u}\right)$ at 4 to 6 weeks of age and approximately $20 \mathrm{~g}$ were purchased from Envigo (Kent, WA) or the Animal Resources Centre (Perth, Australia). Animals were housed in a barrier facility with food and water provided ad libitum on a 12-h light/dark cycle. All applicable international, national, and/or institutional guidelines for the care and use of animals were followed. All experiments were performed with approvals from either The Fred Hutchinson Cancer Research Centre Institutional Animal Care and Use Committee, Telethon Kids Institute Animal Ethics Committee or The University of Queensland Molecular Biosciences committee (IMB/386/18).

\section{Medulloblastoma PDX mouse models}

Studies were conducted using four PDX MB models, Med-211FH, Med-411FH and Med-1712FH and BT084. Med-211FH, Med-411FH and Med-1712FH were generated in the Olson laboratory (Fred Hutchinson Cancer Research Center, Seattle) using paediatric patient tumour tissue obtained from Seattle Children's Hospital with approval from the Institutional Review Board. Med$211 \mathrm{FH}, \mathrm{Med}-411 \mathrm{FH}$ and Med-1712FH are publicly available from https://research.fredhutch.org/olson/en/btrl. html, with details of these models published [25]. Med$211 \mathrm{FH}$ is a $M Y C$-amplified Gp3 MB with classic morphology that was derived from a 2.8-year-old male patient. Med-411FH is a $M Y C$-amplified Gp3 MB with large cell/anaplastic morphology that was derived from a 3year-old male patient. Med-1712FH is a SHH MB with desmoplastic/nodular morphology, derived from a 4.9year-old patient. BT084 PDX model represents SHH MB harbouring TP53 mutation and was gifted from Stefan Pfister (DKFZ, [unpublished]). BT084 cells were modified by lentiviral infection to drive expression of luciferase and green fluorescent protein (GFP) using the pCL20-MSCV-GFP-ires-Luc2 construct kindly provided by Dr Richard Williams of St Jude Children's Research Hospital (Memphis, USA). PDX lines were generated by implanting tumour cells directly into the cerebellum of immunocompromised mice (NSG or athymic nude) within hours of surgical removal from the patient, and then propagating them from mouse to mouse exclusively without in vitro passaging as previously described [25]. Xenografted tumours were subjected to genomic analysis and compared to the primary tumour from which they originated [25].

\section{Subcutaneous and orthotopic xenografts}

To generate subcutaneous tumours, tissue was harvested from intracranial tumours in symptomatic donor mice and immediately processed for transplant into the subcutaneous space of the flank in NSG mice. Tissue was processed in serum-free Dulbecco's Modified Eagle's Medium (DMEM) or phosphate buffered saline (PBS) by trituration through an 18-g or 21-g needle to generate a single cell suspension. The suspension was filtered, centrifuged and re-suspended in 1:1 serum-free DMEM: Matrigel solution to a concentration of $15,000 / \mu l$. One hundred microliters of the tumour cell suspension $(1.5 \times$ $10^{6}$ cells) was injected into the subcutaneous space of the flank of NSG mice.

For orthotopic xenografts, athymic nude mice or NSG were anesthetized and a small incision was made in the skin to expose the skull. A handheld microdrill with a $0.7-\mathrm{mm}$ or $0.9-\mathrm{mm}$ burr was used to create a hole in the calvarium situated above the right cerebellar hemisphere, $2 \mathrm{~mm}$ lateral (right) to the sagittal suture, $2 \mathrm{~mm}$ posterior of the lambdoid suture. Two microliters of cell suspension $(100,000$ cells) in either DMEM (Med-211FH and Med411-FH) or Matrigel (BT084) was injected into the brain parenchyma approximately $2 \mathrm{~mm}$ under the dura. Surgifoam was inserted into the burr hole site. The incision was closed with tissue glue.

\section{Systemic chemotherapy}

Ixabepilone (BMS-247550, Selleckchem) was used for all experiments. Ixabepilone was dissolved in $2 \%$ DMSO, 30\% PEG300, 2\% Tween- 80 and administered by intravenous injection every 4 days for three doses (q4d3) for a total of two cycles, with 2 weeks off in between cycles.

\section{In vivo efficacy studies-subcutaneous tumours}

Studies were conducted using Med-211FH, Med-411FH and Med-1712FH subcutaneous tumours between 200 and $500 \mathrm{~mm}^{3}$ in size. Tumour volume measurements were taken biweekly and mouse weights measured daily. Tumours were measured with calipers, and tumour volume was calculated using the equation: (length $\times$ width $\left.^{2}\right) / 2$ ). Mice were stratified into groups according to tumour size and the groups were randomly assigned by rolling enrolment to therapeutic treatment. Treatment was administered for 30 days or until tumour size reached maximum size requiring ethical collection. 
Changes in tumour volume for each treatment group were reported based upon tumour measurements on day 30 relative to day 1 of treatment. Tumour measurements from mice bearing Med-211FH, Med-411FH and Med1712FH PDX flank tumours treated with ixabepilone or vehicle were analysed using linear mixed models analysis in R. A subset of additional tumour bearing mice were established as described above for assessing the mechanism of tumour regression in response to ixabepilone. For this analysis, drug-treated tumours were collected approximately $5-10 \mathrm{~h}$ after the second dose of ixabepilone whereby tumours had demonstrated evidence of regression and compared to matched vehicle-treated mice at the same timepoint. Tumours were preserved by paraformaldehyde fixation and processed for immunoanalysis.

\section{In vivo efficacy studies-orthotopic tumours}

Orthotopic xenotransplantation was performed in athymic nude mice for Med-211FH and BT084 or NSG mice for Med-411FH. For Med-411FH, tumour growth was confirmed in each mouse by the onset of symptoms and randomly assigned into treatment groups. For Med-211, tumour growth was confirmed by the onset of symptoms and the cohort of mice were stratified according to the severity of symptoms and randomly assigned into treatment groups. For BT084, tumour growth was monitored weekly by bioluminescence using a Lago X small animal imager (Spectral Instruments Imaging, USA) and treatment commenced once bioluminescence reached approximately $5 \times 10^{5}$ photons/second. Mice enrolled in the study were observed daily and weights recorded daily (Med-411FH) or three times per week (Med-211FH and BT084). Euthanasia was performed prior to the end of the study if mice demonstrated signs of tumour-related morbidity or lost more than $20 \%$ body weight loss. Kaplan-Meier survival estimates were calculated using GraphPad Prism 7.0a software, and survival curves are presented in results. Differences in survival between groups were performed using Log rank (Mantel Cox) test. Median survival and 95\% confidence interval (95\% CI) are reported.

\section{Immunoblotting analysis of PDX tumours}

Whole cell extracts from MB PDX were prepared using radioimmunoprecipitation assay buffer (RIPA) buffer $(150 \mathrm{mM} \mathrm{NaCl}, 1 \%$ Nonidet P-40, $0.5 \%$ sodium deoxycholate, $0.1 \%$ SDS, $25 \mathrm{mM}$ Tris $\mathrm{pH} 7.4$ ) with protease and phosphatase inhibitors (Cell Signalling, 5872). Total protein concentrations were determined using the BCA kit (Pierce, 23225). Equal amounts of total protein extract were resolved on SDS-PAGE and transferred to a polyvinylidene difluoride membrane using a transfer apparatus according to manufacturer's protocols (Invitrogen, B1000). After incubation with 5\% non-fat milk in
TBST (10 mM Tris, pH 7.6, $150 \mathrm{mM} \mathrm{NaCl,} \mathrm{0.1 \%} \mathrm{Tween}$ 20) for $60 \mathrm{~min}$, the membrane was washed once with TBST and incubated with antibodies against: TUBB3 (Covance, MRB-435P, 1:1000) and Beta-actin (BACT) (abcam, ab8224, 1:500) at $4^{\circ}$ for 16-18 h. Membranes were washed three times for five minutes and incubated with a 1:2500 of horseradish peroxidase-conjugated antimouse or anti-rabbit secondary antibody (1:2500) for $1 \mathrm{~h}$ at room temperature. Blots were washed with TBST three times and developed using the ECL system (Amersham Biosciences) according to manufacturer's protocol.

\section{Immunofluorescence analysis of PDX tumours}

Antibody markers were analysed on seven micron, paraffin-embedded sections via standard immunofluorescence techniques using the following antibodies: Phospho-Histone H3 (pHH3) (Cell Signalling \#9701, 1: 100), marker of proliferation (Ki67) (BD Pharmingen \#556003, $2.5 \mu \mathrm{g} / \mathrm{ml}$ ) and cleaved caspase-3 (CC3) (abcam \#ab2302, $10 \mu \mathrm{g} / \mathrm{ml})$. High-temperature unmasking was performed with preheated $\mathrm{pH} 6.0$ citrate buffer (Vector Labs) for $5 \mathrm{~min}$, and mouse-on-mouse blocking reagent was used to block non-specific binding of mouse primary antibodies (Vector Labs). For the quantitation of positively staining pHH3, CC3 and/or Ki67 cells: tumour cell nuclei as identified by DAPI were scored for the presence or absence of $\mathrm{pHH} 3, \mathrm{CC} 3$ and Ki67 staining in five fields of view per tumour in triplicate for each treatment group [26]. GraphPad Prism 7.0a software was used to analyse immunofluorescence difference between treatment groups using a Welch's $\mathrm{t}$ test for $\mathrm{pHH} 3$ and a Holm-Sidak corrected multiple $t$ test for the CC3 and Ki67 co-stain.

\section{Results}

Regulatory networks identified in a MB mouse model discover novel drug candidates common to all human non-WNT MB

To discover novel drugs with potential anti-tumour activity in human $\mathrm{MB}$, we identified differentially expressed genes common to all non-WNT MB $(P<$ 0.05), namely SHH, Gp3 and Gp4 MB, and mapped expression values to the functionally derived protein interaction network we have described previously [16]. Of the 168 significantly differentially expressed genes, 84 were significantly over-expressed in all three MB subgroups, $\mathrm{SHH}, \mathrm{Gp} 3$ and Gp4, and are subsequently referred to here as the commonly over-expressed genes for non-WNT MB. A refined PPI network was then created including only the proteins from these commonly overexpressed genes and their direct interaction partners (Fig. 1, Additional file 4: Table S3). To identify the druggable components of this network, drugs with known protein targets were mapped to the network, generating 


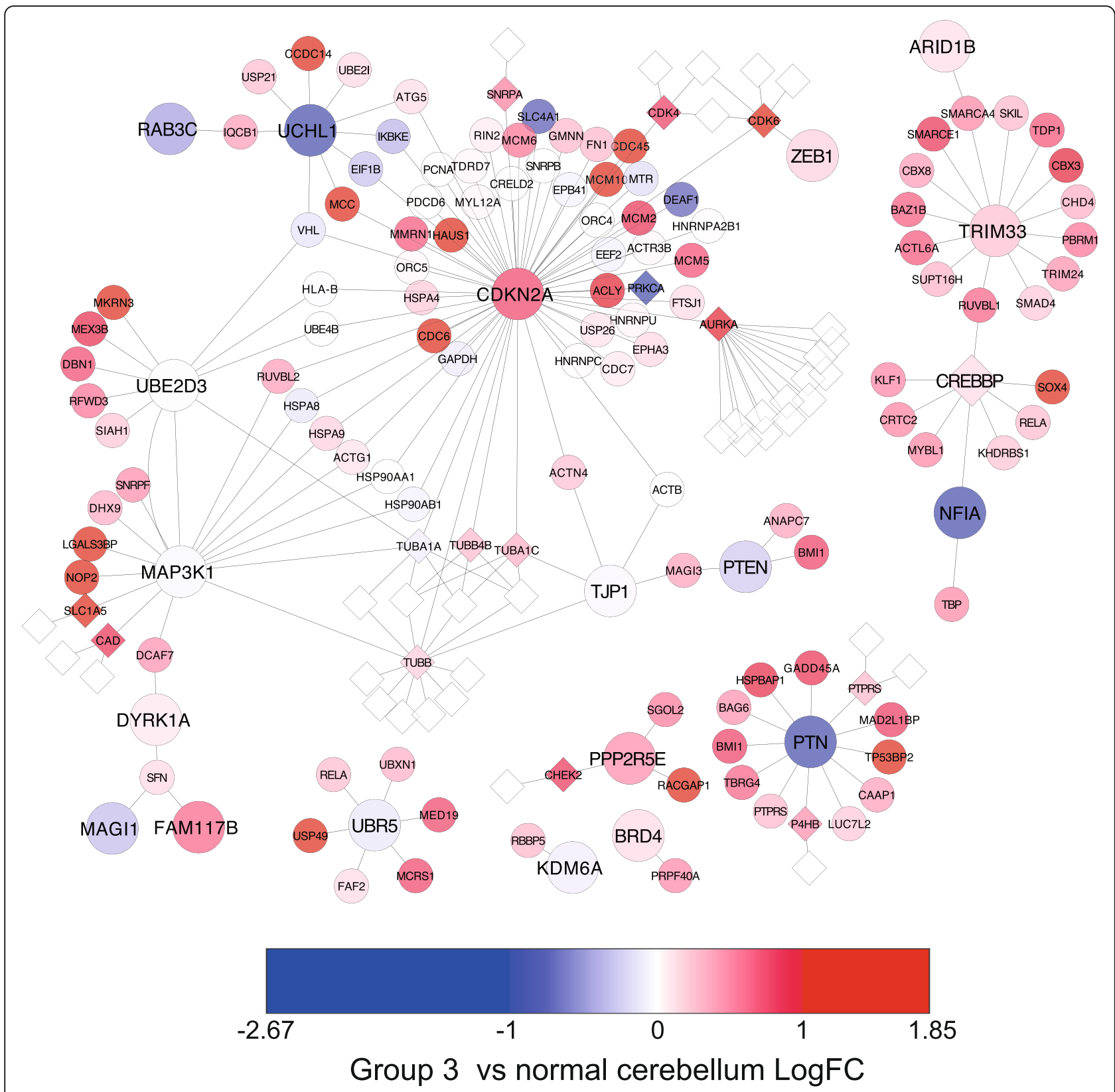

Fig. 1 Local PPI network representing the druggable proteins for non-WNT MB. CIS-derived candidate cancer genes are represented by large circles, whilst small circles represent the proteins that interact with these CIS-derived candidates. Nodes coloured in red-light red represent significantly over-expressed proteins in Gp3 MB, whilst blue-light blue nodes represent significantly under-expressed proteins in Gp3 MB. White small diamond shaped nodes represent drugs for that protein. For expression data for each node in the network, see Additional file 4

a drug-target network containing 145 proteins and 36 drugs. The network contains one large connected component, with several smaller components disconnected from the main part of the network (Fig. 1). The central component of the network contains proteins derived from multiple genes that we had previously identified through our functional screen as drivers of $\mathrm{MB}$ growth: cyclin-dependent kinase inhibitor 2A (CDKN2A), zinc finger E-Box binding homeobox 1 (ZEB1), tight junction protein 1 (TJP1), mitogen-activated protein kinase kinase kinase 1 (MAP3K1), family with sequence similarity 117 , member B (FAM117B), dual-specificity tyrosine-(Y)phosphorylation regulated kinase 1A (DYRK1A), ubiquitin-conjugating enzyme E2D 3 (UBE2D3), ubiquitin C-terminal hydrolase L1 (UCHL1), member RAS oncogene family (RAB3C), phosphatase and tensin homologue (PTEN), membrane-associated guanylate kinase, WW and PDZ domain (MAGI1) and ubiquitin 
protein ligase E3 component N-recognin 5 (UBR5) [16]. Seven over-expressed genes identified as druggable interact with the CIS-derived candidate cancer proteins residing in this central component of the network (Table 1). Five of these, AURKA, CDK4, CDK6, small nuclear ribonucleoprotein polypeptide A (SNRPA) and TUBB, directly interacted with CIS CDKN2A, suggesting the therapeutic targeting of any of these druggable proteins may influence functions associated with CDKN2A. The MAP3K1 hub contains three significantly over-expressed proteins that are druggable: TUBB, carbamoyl-phosphate synthetase 2, aspartate transcarbamylase, and dihydroorotase (CAD) and solute carrier family 1 (neutral amino acid transporter) member 5 (SLC1A5). The identification of multiple significantly over-expressed druggable proteins interacting with CDKN2A and MAP3K1 further emphasizes the role for both of these proteins not only in driving accelerated MB tumorigenesis [16], but also as potential foci of targeted therapies.

In addition to the potential for combinatorial targeting of CDKN2A and MAP3K1, two over-expressed druggable proteins residing within this central component of the network were found to interact with more than one CIS-derived candidate cancer protein. CDK6 interacted with both CDKN2A and ZEB1, and as noted above, TUBB interacts with both MAP3K1 and CDKN2A. CDK6 and TUBB therefore act as network "bridging nodes", that is, nodes that connect otherwise separate regions of the network and represent proteins through which previously identified candidate cancer genes driving $\mathrm{MB}$ development may exert common effects. Proteins with topological properties such as this represent likely points of cross-talk and are highly attractive therapeutic targets [29]. Whilst the above-mentioned analysis identified potential therapeutic targets common to all non-WNT MB, we also performed drug-target

Table 1 Significantly over-expressed druggable network proteins and their associated CISs common to all non-WNT MB

\begin{tabular}{lll}
\hline Network protein & CIS & $\begin{array}{l}\text { Evidence as a novel } \\
\text { therapeutic target } \\
\text { for } \text { MB }\end{array}$ \\
\hline AURKA & {$[1,2]$} \\
CAD & CDKN2A & \\
CDK4 & MAP3K1 & {$[3-6,8]$} \\
CDK6 & CDKN2A & {$[3-6,8]$} \\
CHEK2 & CDKN2A/ ZEB1 & {$[9]$} \\
P4HB & PPP2R5E & \\
PTPRS & PTN & \\
SLC1A5 & PTN & {$[16]$} \\
SNRPA & MAP3K1 & \\
TUBB & CDKN2A & {$[27,28]$} \\
\hline
\end{tabular}

network analysis on individual molecular sub-groups of $\mathrm{MB}$, identifying a list of novel drug candidates likely to target that specific subgroups of $\mathrm{MB}$ (Additional file 5: Fig. S2).

\section{Targeting microtubule dynamics induces significant regression of $\mathrm{Gp} 3$ and $\mathrm{SHH}$ patient-derived $\mathrm{MB}$}

TUBB emerged as the leading candidate based on its highly attractive network topology and pharmacogenomic properties. Epothilone B agents are a class of new generation microtubule-stabilizing agents (MSA) that bind to the $\beta$-tubulin subunit of microtubules. Of the three Epothilone B agents, patupilone, ixabepilone and sagupilone, ixabepilone is the most mature analogue in development, with published phase II and III data and regulatory approval for clinical use in the treatment of breast cancer [30]. On this basis, ixabepilone was selected for the targeting TUBB in this manuscript.

To investigate the anti-tumour efficacy of ixabepilone, we used well established PDX models of MB which are strictly maintained in vivo and shown to maintain the characteristics of the human primary tumours from which they were derived in terms of histology, immunohistochemistry, gene expression, DNA methylation, copy number and mutational profiles [25]. We analysed the results from previous microarray analyses of MB PDX models to evaluate expression of TUBB in our cohort [25]. High mRNA expression of TUBB was confirmed in a range of MB PDX including Med-1712FH (SHH), Med211FH (Gp3), Med-411FH (Gp3) and Med-813FH (SHH) (Fig. 2a), consistent with the network analysis of human MB (Fig. 1). Given tubulin expression is predominantly regulated post-transcriptionally and transcript levels do not necessarily reflect protein levels [31, 32], we defined tubulin protein levels in PDX lysates by western blot. Strong expression of beta isotype $\beta$ III (TUBB3) was confirmed in all MB PDX (Fig. 2b).

Med-211FH and Med-411FH PDX cells were implanted in the flank of mice and animals with established tumours were treated with vehicle or ixabepilone. Tumour growth was significantly reduced in drugtreated mice, with all Med-211FH $(P<0.001$, Fig. 2c) and Med-411FH $(P<0.001$, Fig. $2 \mathrm{~d})$ tumours exhibiting complete regression prior to completion of ixabepilone treatment, with no palpable tumour detected by day 19 and day 22 for Med-211FH and Med-411FH respectively. In contrast, growth continued at a high rate in the vehicle-treated tumours, increasing in size by approximately 5-6-fold for Med-211FH (Fig. 2c) and 5.62-fold for Med-411FH (Fig. 2d) before tumours reached maximum size requiring collection.

Whilst Med-211FH and Med-411FH are both models of Gp3 MB, our analyses predicted TUBB as a therapeutic candidate for multiple subgroups of $\mathrm{MB}$, including $\mathrm{SHH}$. 


\section{a}

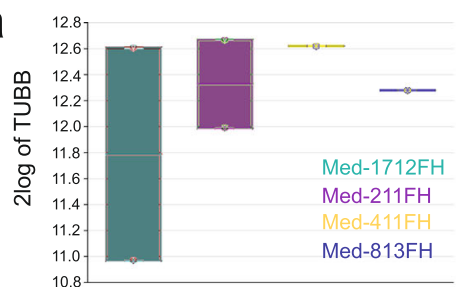

C

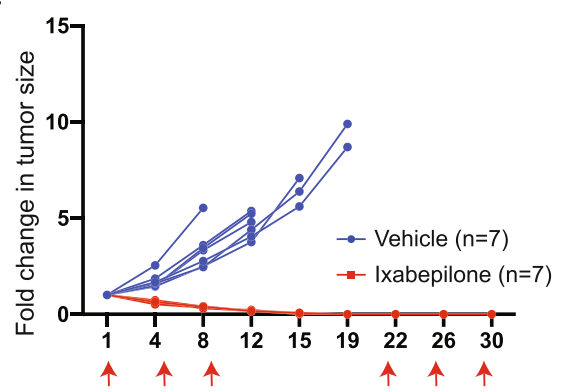

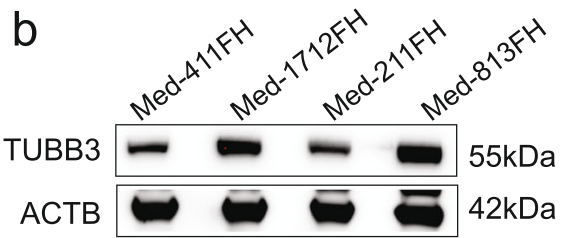

d

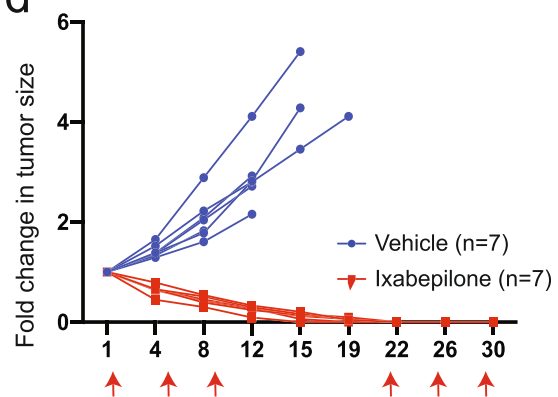

e
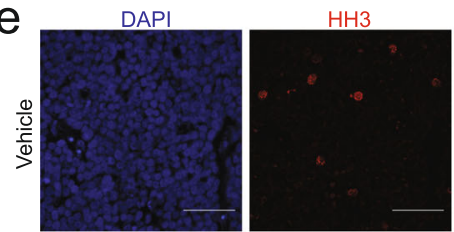

Merged
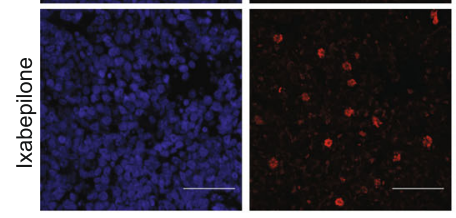

f
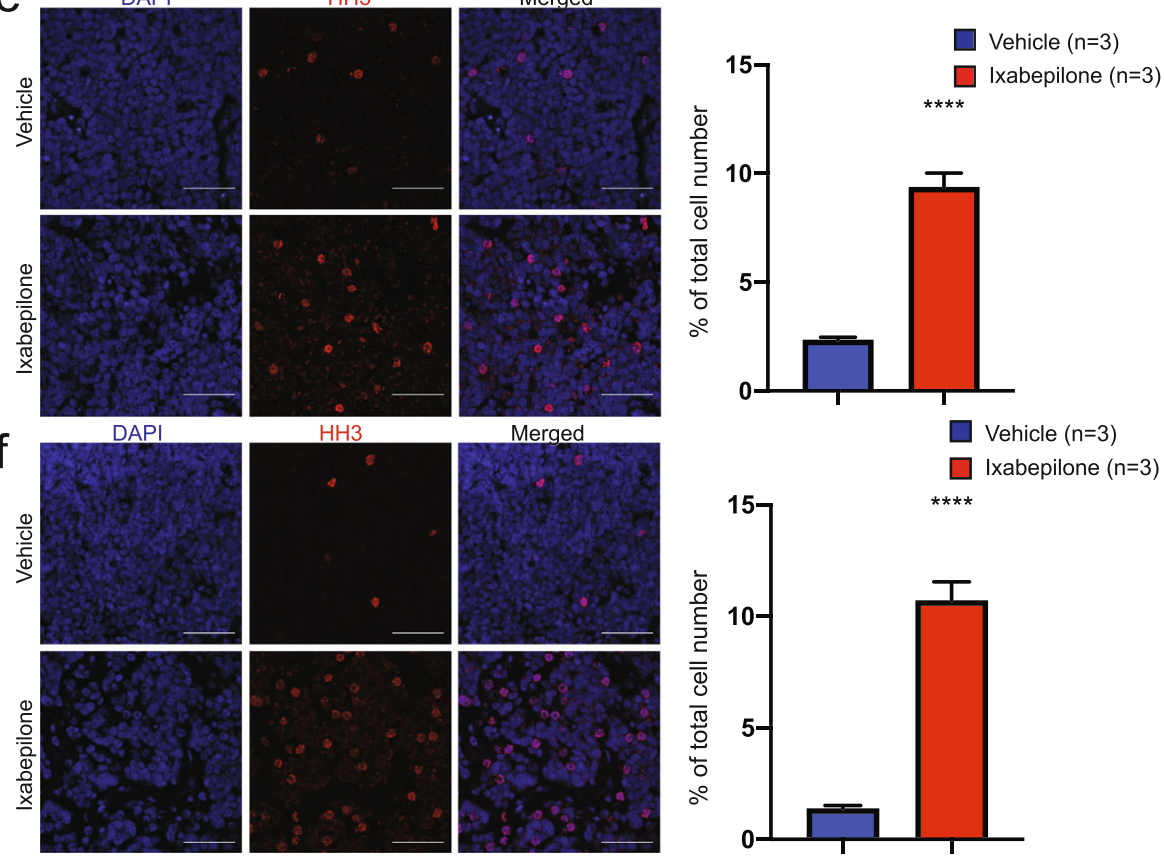

Fig. 2 Ixabepilone causes regression of MYC-amplified Gp3 MB. a Relative mRNA expression of TUBB in Med-1712FH, Med-211FH, Med-411FH and Med-813FH PDX as compared to other paediatric brain tumours (PDX, matching human primary brain tumours and cell lines) using the R2 Genomics Analysis and Visualization Platform (http://r2.amc.nl). b Immunoblot analysis of TUBB3 and ACTB levels in Med-411FH, Med-1712FH, Med-211FH and Med-813FH PDX. Blots were scanned using BioRad ChemiDoc MP Imager and cropped using Adobe Illustrator. Fold change in tumour volume following treatment with ixabepilone or vehicle in mice bearing two Grp3 PDX models, c Med-211FH and d Med-411FH subcutaneous tumours. Tumour measurements were compared between vehicle and drug treatments using linear mixed models analysis in R. Treatment days are indicated by a red arrow on each graph. Representative images of $\mathrm{HH} 3$ immunostaining as a marker of mitosis respectively in Med-211FH (e, left) and Med$411 \mathrm{FH}$ (f, left) subcutaneous tumours following vehicle (top panel) and ixabepilone (bottom panel). Scale bar $20 \mu \mathrm{m}$. Quantitative analysis of HH3 staining in Med-211FH (e, right) and Med-411FH (f, right) tumours. Percentage of tumour cells staining positive were quantified for vehicle ( $n=3$ ) and drug-treated tumours $(n=3)$ using Image J software. Data are presented as the mean \pm SEM. Statistical evaluation was performed using a Holm-Sidak corrected multiple t test with statistically significant differences indicated $(* P<0.05, * * * P<0.0001)$

On this basis, Med-1712FH PDX cells were implanted in the flank of mice and animals with established tumours were treated with vehicle or ixabepilone. Similar to the previous Gp3 PDX models, tumour growth was significantly reduced in drug-treated mice, with an average reduction in tumour volume of $67.61 \%(P<0.001$, Additional file 6: Fig. S3) whilst vehicle-treated tumours increased in size by approximately 2.47 -fold. Together, these 
data serve as powerful validation of our strategy, demonstrating for the first time that the targeting of TUBB via microtubule stabilizer ixabepilone is an efficacious therapeutic approach for multiple subgroups of $\mathrm{MB}$.

\section{In vivo anti-tumour activity of ixabepilone is associated} with mitotic arrest, decreased cell proliferation and the induction of apoptosis

Ixabepilone binds to the $\beta$-tubulin subunit of microtubules, inducing microtubule polymerization and subsequent $\mathrm{G} 2 / \mathrm{M}$ arrest, decreased proliferation and the induction of apoptosis [33-36]. On this basis, we sought to validate the mechanism of action and anti-tumour effects of Ixabepilone in vivo by immunofluorescence using phosphorylated histone $\mathrm{H} 3(\mathrm{pHH} 3)$ as a specific marker of mitosis, CC3 as a marker of apoptosis and Ki67 as a marker for proliferation. Med-211FH and Med-411FH PDX cells were implanted in the flank of mice and tumours were resected after just two drug administration doses. Ixabepilone demonstrated impressive single agent efficacy after 5 days (two doses), with an average reduction in tumour volume of $38.62 \%$ for Med211FH (Fig. 2c) and 29.37\% for Med-411FH (Fig. 2d).

Immunofluorescence analysis of drug-treated tumours showed a clear increase in the number of cells of drugtreated tumours in mitosis, with quantitative analysis indicating an increase of approximately $7 \%$ and $9 \%$ of pHH3 positive cells in drug-treated Med-211FH $(P<0.001$, Fig. $2 \mathrm{e})$ and Med-411FH $(P<0.001$, Fig. $2 \mathrm{f})$ tumours respectively. All drug-treated tumours of both Med-211FH and Med-411FH PDX also displayed decreased proliferation and increased apoptosis as compared to vehicle-treated tumours (Fig. 3). Quantitative analysis indicated drugtreated tumours displayed a reduction of approximately $26 \%$ and $32 \%$ of Ki67 positive cells in Med-211FH $(P<$ 0.001 , Fig. 3a right $)$ and Med-411FH $(P<0.001$, Fig. 3b right) respectively, with a concomitant increase of approximately $3 \%$ and $11 \%$ of CC3 positive cells in Med-211FH $(P=0.013$, Fig. 3a right $)$ and Med-411FH $(P<0.001$, Fig. 3b right) respectively. These data validate the hypothesis that the specific inhibition of microtubule dynamics via ixabepilone has significant in vivo antitumour effects in Gp3 PDX MB by arresting cells at mitosis, suppressing proliferation and inducing apoptosis.
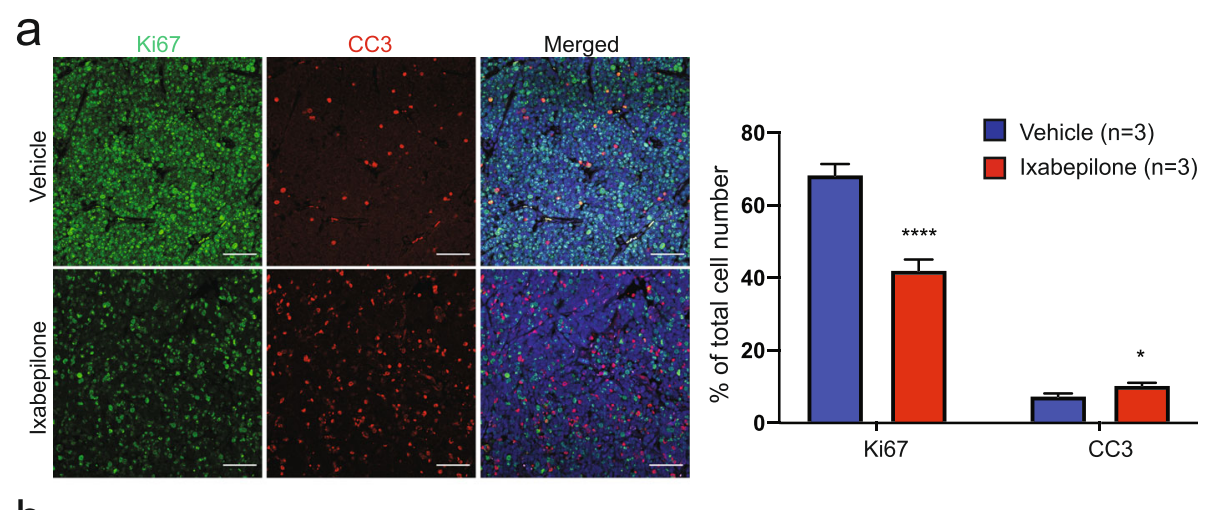

b
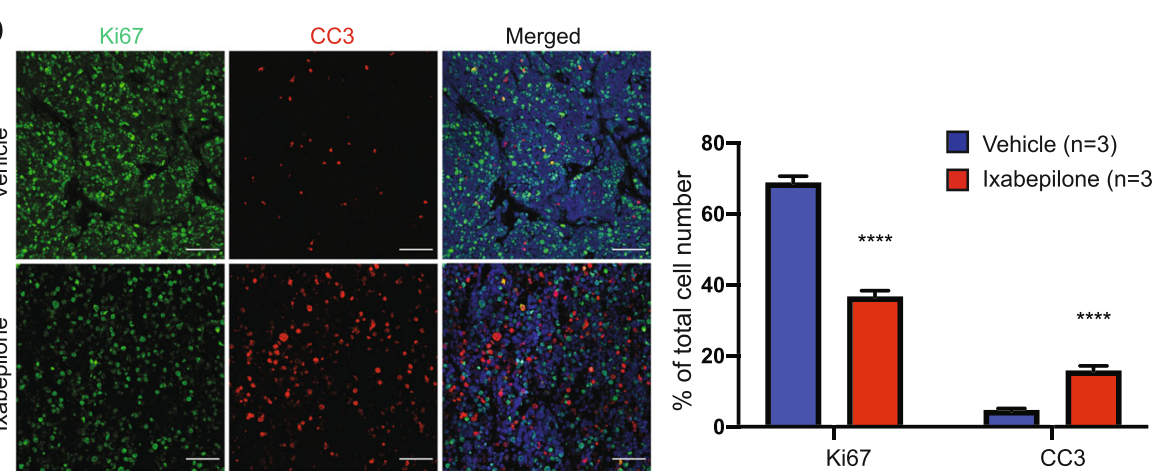

Fig. 3 Ixabepilone induces apoptosis and decreases proliferation in MYC-amplified Gp3 MB. a Representative images of Ki67 and CC3 immunostaining as a marker of cell proliferation and apoptosis respectively in Med-211FH (a, left) and Med-411FH (b, left) subcutaneous tumours following vehicle (top panel) and ixabepilone (bottom panel). Scale bar $20 \mu \mathrm{m}$. Quantitative analysis of Ki67 and CC3 staining in Med-211FH (a, right) and Med-411FH (b, right) tumours. Percentage of tumour cells staining positive were quantified for vehicle ( $\mathrm{n}=3$ ) and drug-treated tumours ( $n=3$ ) using Image J software. Data are presented as the mean \pm SEM. Statistical evaluation was performed using a Holm-Sidak corrected multiple $t$ test with statistically significant differences indicated $\left.{ }^{*} P<0.05,{ }^{* * *} P<0.0001\right)$ 
Ixabepilone treatment significantly extends the survival of mice engrafted with Gp3 and SHH MB PDXs

We then investigated the efficacy of ixabepilone in the treatment of MYC-amplified PDX established as intracranial tumours in mice, previously demonstrated as the gold standard in the field of brain cancer modelling [37]. Mice bearing intracranial Med-211FH and Med-411FH tumours were enrolled and treated with ixabepilone or vehicle. Drug-treated animals showed a significant increase in survival for both Med-211FH (Fig. 4a, median survival $=72$ days post treatment, $95 \% \mathrm{CI} 60$-infinity for treatment, 45 days post treatment $95 \%$ CI 14-infinity for vehicle, $P=0.016$ ) and Med-411FH (Fig. $4 \mathrm{~b}$, median survival $=11$ days post treatment $95 \%$ CI 8 to infinity for treatment vs. median survival $=5.5$ days post treatment 95\% CI 3-infinity for vehicle, $P=0.022$ ). For Med$211 \mathrm{FH}$, the probability of survival in the treatment group at 60 days post-treatment was $62.5 \%$ (95\% CI 36.54-100) and $14.29 \%$ in the vehicle group (95\% CI 2.33-87.69). For Med-411FH, the probability for survival in the treatment group at 30 days post-treatment was $25 \%$ (95\% CI 9.38-66.61) and 0\% in the vehicle group (95\% CI 0-0).

In a follow-up study, mice bearing orthotopic BT084 $\mathrm{MB}$ of the SHH subgroup (subtype alpha), which express a detectable signal of luciferase by bioluminescence, were treated with vehicle or ixabepilone. Drug-treated animals showed a significant increase in survival (Fig. 4c) where the probability for survival in the treatment group at 40 days post-treatment was $66.67 \%(95 \% \mathrm{CI}$ $42-100$ and $12.5 \%$ in the vehicle group (95\% CI 278.19). Weekly evaluation of tumour growth by bioluminescence showed a significant reduction in tumour size in drug-treated animals as confirmed by the smaller increase in total photo flux from luciferase expressing BT084 xenografts (Fig. 5a).

Asymptomatic drug-treated mice bearing orthotopic BT084 MB were collected at 171 days post-treatment for histologic examination, with minimal tumour burden (Fig. 5b) observed compared to large tumours in vehicletreated mice at 29 days (Fig. 5c). These results clearly demonstrate ixabepilone significantly prolonged survival of mice bearing intracranial tumours by dramatically reducing tumour size. Taken together, our findings confirm that the targeting of TUBB using a microtubule stabilizing agent is a viable therapeutic avenue for Group 3 and $\mathrm{SHH} \mathrm{MB}$, with these data representing biological validation of the predictive power of our systems pharmacogenomics approach to discover and validate novel drug candidates for all non-WNT MB.

\section{Discussion}

Systems pharmacogenomics is a powerful approach for the discovery of novel druggable targets in cancer, with previous studies successfully using this approach in both

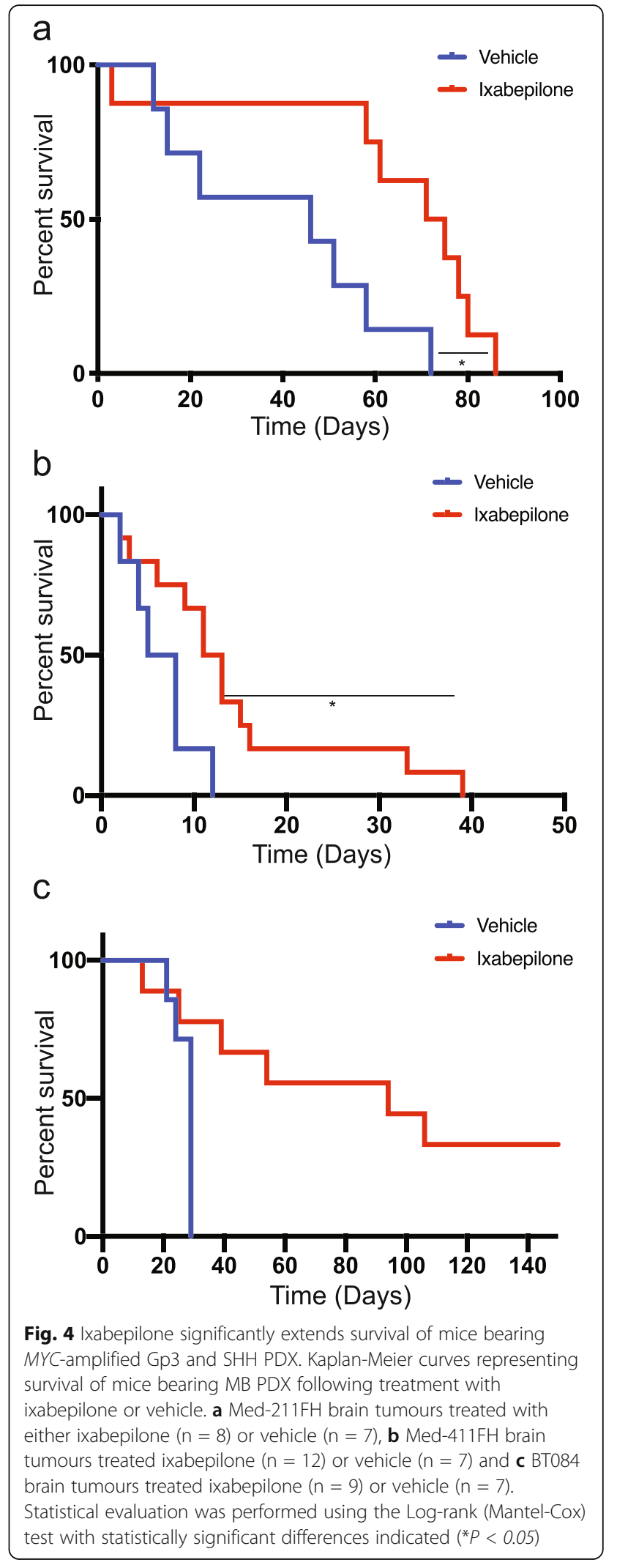


a

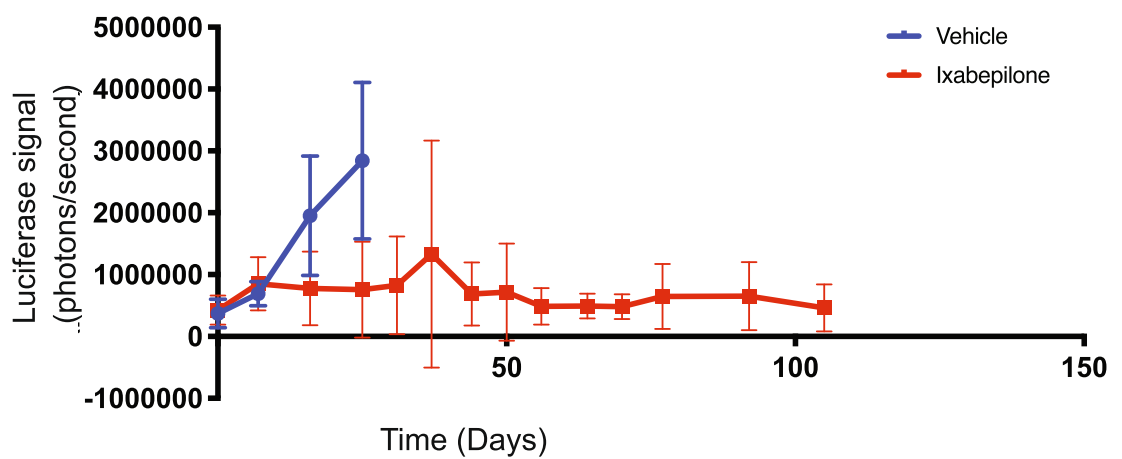

b
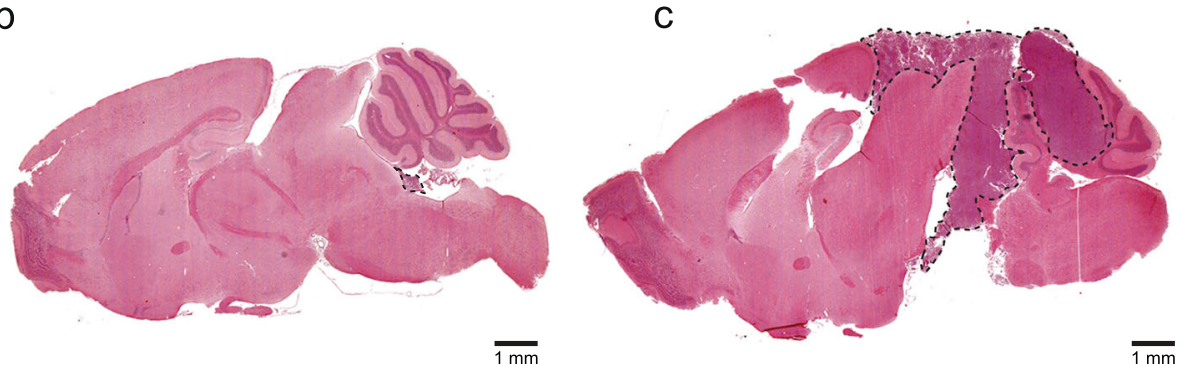

Fig. 5 Ixabepilone causes dramatic reduction in tumour growth in orthotopic sonic hedgehog (SHH) medulloblastoma. a Tumour growth was monitored by bioluminescence following treatment with ixabepilone or vehicle in mice bearing BT084 SHH orthotopic tumours. Tumour measurements were compared between vehicle and drug treatments using linear mixed models analysis in R. Treatment days are indicated by vertical lines on the graph. Representative $\mathrm{H}$ and $\mathrm{E}$ images of ixabepilone-treated mice (b) at 171 and vehicle-treated mice (c) at 29 days posttreatment. Scale bar $1 \mathrm{~mm}$

in vitro and in vivo models to functionally predict and validate pharmacogenomic candidates [27, 28, 38-40]. Large scale genomic and transcriptomic analyses of $\mathrm{MB}$ have broadened our understanding of the molecular and genetic features driving $\mathrm{MB}$ development $[2,3,5,41]$.

Our increasing knowledge of these features has enabled the identification of a number of candidate genes as potential therapeutic targets for certain subgroups of this disease $[18,19,42-44]$ and our own prior work confirmed a large number of genes functionally shown to drive tumorigenesis. However, methods to integrate the large amounts of data obtained from several mouse and human MB model analyses to the discovery and prioritization of novel drug targets for pre-clinical screening are still urgently required.

In this study, we have described a functional pharmacogenomics and network-driven systems biology approach based upon candidate cancer-promoting genes identified from our previously published $S B$ mouse model of MB [16]. Whilst we could have expanded our network to include genes such as those identified in previous genomic studies [41, 45, 46], we chose to focus on the use of the CIS-derived cancer genes and the broader network to select for these novel therapeutic targets using the functional evidence of the mutagenesis screen as a guide. Many of these CIS-derived cancer genes are known to be mutated in human $\mathrm{MB}$, as are other genes that interact with these in our network (Additional file 7: Table S4). The network itself is built using only experimentally verified PPI manually curated from the literature, and when combined with functional hits from the genetic screen, and expression data from patients, represents a powerful discovery tool. Integrating our functionally derived network with gene expression data from a large cohort of patient samples, we identify multiple proteins targeted by existing drugs that can be evaluated as potential MB therapeutics. This approach identified many confirmed targets with existing drugs that are currently used in the clinic or are in various stages of clinical trials in other cancers (for example clinical trials for drugs targeting AURKA and BRD4), emphasizing the efficacy of our approach $[18,19,21$, 47-50]. We previously validated CDK4/6 inhibition as a successful therapeutic approach for both Gp3 and $\mathrm{SHH}$ MB [20], consistent with an additional study demonstrating the efficacy of palbociclib as a single agent in the treatment of $M Y C$-driven $\mathrm{MB}$ in an in vitro cerebellar human neural stem cell model of MB [51].

TUBB emerged as a leading candidate from our network analysis. Targeting TUBB via ixabepilone in four PDX models confirms this new generation MSA as an effective therapy for aggressive $\mathrm{MB}$ and validates an 
additional target from our functional network, demonstrating its power for the discovery of new MB therapies through drug repositioning. Significantly over-expressed druggable targets, such as TUBB, MYC, SMAD2, RARA and CDK6 for Gp3 MB, represent highly attractive therapeutic candidates due to their expression and centrality in the network, connected as they are to multiple driver genes [16]. Pharmacologic inhibition of these proteins has the potential to elicit both local and widespread global effects on the biological system sustaining $M B$, thereby stimulating a greater therapeutic response via simultaneous targeting of multiple biological pathways encompassed within these networks.

Microtubule targeting agents (MTAs) have been heavily exploited as anticancer agents, with certain classes of MTAs approved for several adult cancer types including breast, ovarian, lung, oesophageal, endometrial, cervical, prostrate and head and neck cancers [30, 52]. MTAs comprise many chemically diverse compounds which can be broadly classified into microtubule stabilizing agents, such as the taxanes and epothilones, and microtubule-destabilizing agents such as the Vinca alkaloids (reviewed in [49]). First-generation taxanes such as Paclitaxel and Docetaxel are high-affinity substrates for several $A B C$ drug efflux transporters [50, 51]. Given the expression of these has been reported in a variety of central nervous system tumours including $\mathrm{MB}[52,53]$, first-generation taxanes are expected to have limited efficacy in $M B$ and were therefore not selected for this study.

Vincristine, a microtubule destabilizing agent specifically targeting TUBB and tubulin alpha- $4 \mathrm{~A}$ chain (TUBA4A), is a chemotherapeutic currently used for the management of standard-risk and high-risk MB [53]. However, due to its large size (MW 825 Daltons) and susceptibility to transport by ATP-binding cassette $(\mathrm{ABC})$ drug efflux transporters such as P-glycoprotein (P-gp, encoded by the gene $M D R-1 / A B C B 1$ gene) and multidrug resistance protein (MRP1, encoded by the $M R P 1 / A B C C 1$ gene) [54-56], vincristine does not penetrate well into brain tumour tissue. We have previously shown cabazitaxel, a second-generation taxane of the microtubule stabilizing class of MTAs, significantly extended survival of mice bearing orthotopic Med-211FH PDX tumours compared to vehicle controls [57]. Whilst these results were promising, growing clinical evidence indicates high expression of TUBB3 as a clinical biomarker of taxane resistance in various tumour types (reviewed in [32]), but has been shown to predict sensitivity to epothilone B compounds [58, 59]. Given the strong expression of TUBB3 in PDX models of MB observed in this study, epothilone B agents may be the preferred MTA and give rise to an overall more durable response. Semi-synthetic Epothilone B agent patupilone was previously found to reduce the proliferative capacity and clonogenicity in vitro of a variety of MB cell lines and delayed the growth of the D425 MB cell line in vivo following subcutaneous implantation [60]. The impressive efficacy of ixabepilone (Figs. 2, 4 and 5), an additional semi-synthetic second-generation Epothilone B compound, strongly highlights the potential of MSA for the treatment of children diagnosed with MB. Functional studies investigating the role of $\beta$-tubulin isotypes in sensitivity and resistance to epothilones in $\mathrm{MB}$ is required and is anticipated to facilitate the identification of biomarkers to accurately select $\mathrm{MB}$ patients most likely to respond to MTAs.

\section{Conclusions}

The in vivo validation we present here for targeting TUBB with ixabepilone and previous validation of CDK6 targeting with palbociclib as two successful therapeutic options for MB support the power of our functionally derived network in identifying candidates and suggests that other players in this network with similar topological and biological properties may also have therapeutic efficacy in MB. Experimentally grounded molecular interaction networks constructed around functionally validated cancer driver genes capture real and significant biological mechanisms that are essential to cancer and can be productively targeted pharmacologically. By integrating extensive molecular profiling of patients as we have done here, such networks present powerful tools for the characterization of therapeutic candidates, the repositioning of drugs and ultimately the design of patientinformed therapies.

\section{Abbreviations}

MB: Medulloblastoma; CDK4: Cyclin-dependent kinase 4; CDK6: Cyclindependent kinase 6; TUBB: Tubulin beta chain; AURKA: Aurora kinase A; WNT: Wingless; CAD: Carbamoyl-phosphate synthetase 2, aspartate transcarbamylase, and dihydroorotase; SNPRA: Small nuclear ribonucleoprotein polypeptide A; SLC1A5: Solute carrier family 1 (neutral amino acid transporter) member 5; PTPRS: Protein tyrosine phosphatase receptor type S; P4HB: Protein disulfide-isomerase; CHEK2: Checkpoint kinase 2; SHH: Sonic hedgehog; Gp3: Group 3; Gp4: Group 4; SMO: Smoothened; PPI: Protein-protein interactions; SB: Sleeping Beauty; Ptch1: Patched 1; CIS: Common insertion sites; PDX: Patient-derived xenograft; CDKN2A: Cyclin-dependent kinase inhibitor 2A; ZEB1: Zinc finger E-box binding homeobox 1; TJP1: Tight junction protein 1; MAP3K1: Mitogen-activated protein kinase kinase kinase 1; FAM117B: Family with sequence similarity 117, member B; DYRK1A: Dualspecificity tyrosine-(Y)-phosphorylation regulated kinase $1 A$;

UBE2D3: Ubiquitin-conjugating enzyme E2D3; UCHL1: Ubiquitin C terminal hydrolase L1; RAB3C: Member RAS oncogene family; PTEN: Phosphatase and tensin homologue; MAGI1: Membrane-associated guanylate kinase, WW and PDZ domain; UBR5: Ubiquitin protein ligase E3 component N-recognin 5; MSA: Microtubule-stabilizing agent; TUBB3: Beta isotype $\beta$ III; pHH3: Phosphorylated histone H3; Ki67: Marker of proliferation Ki-67; CC3: Cleaved caspase 3; MYC: Myc proto-oncogene protein; $\mathrm{Cl}$ : Confidence interval; MTAs: Microtubule targeting agents; TUBA4A: Tubulin alpha-4A chain; ABC: ATP-binding cassette; MRP1: Multidrug resistance protein 


\section{Supplementary Information}

The online version contains supplementary material available at https:/doi. org/10.1186/s13073-021-00920-z.

Additional file 1: Supplementary Figure 1. Schematic of data integration to identify and validate therapeutic targets for MB.

Additional file 2: Supplementary Table S1. Network parameters for subgroup-specific network analysis.

Additional file 3: Supplementary Table S2. Putative drug targets and drugs in subgroup-specific networks.

Additional file 4: Supplementary Table S3. Expression Data associated with Figure 1.

Additional file 5: Supplementary Figure S2. Local protein interaction network representing significantly over-expressed druggable proteins for individual subgroups of MB.

Additional file 6: Supplementary Figure S3. Ixabepilone causes regression of sonic hedgehog $(\mathrm{SHH}) \mathrm{MB}$.

Additional file 7: Supplementary Table S4. Summary of mutational status in human Medulloblastoma of key CIS genes identified in the mutagenesis screen,

\section{Acknowledgements}

The authors would like to thank the patients and families who contributed brain tumour tissue for the PDX models.

\section{Authors' contributions}

L.A.G and M.J.D designed research; L.A.G, M.J.D, A.M, E.T, M.S, E.H., E.G., C.A. and M.K. performed the research; L.A.G., C.B., D.D.B, M.D.T and M.J.D contributed new datasets, reagents and analytical tools; L.A.G., M.J.D., C.A., R.E., E.G., E.H., A.B., D.D.B and M.K. analysed the data: L.A.G., M.J.D, J.M.O., and B.J.W. wrote the paper. All authors read and approved the final manuscript.

\section{Funding}

This work is supported by NIH/NCI RO1 CA112250, NIH/NCI RO1 CA135491 and Seattle Children's Brain Tumour Endowment (E.G and J.M.O), as well as the Kids Cancer Project (B.J.W), Brainchild (B.J.W), Children's Hospital Foundation (L.A.G and B.J.W), The Pirate Ship Foundation (R.E. and N.G.), Cure Brain Cancer Foundation (L.A.G and B.J.W) and Cure Brain Cancer Foundation National Breast Cancer Foundation (M.J.D)

\section{Availability of data and materials}

All gene human gene expression data used in this study are available through the Gene Expression Ombibus at GSE37382. https://www.ncbi.nlm. nih.gov/geo/query/acc.cgi?acc=GSE37382 [41] and GSE167447. https://www ncbi.nlm.nih.gov/geo/query/acc.cgi?acc=GSE167447 [61]. All Bioinformatics software used and cited in this study are open access and freely available.

\section{Declarations}

Ethics approvals and consent to participate

All experimental protocols were approved by either The Fred Hutchinson Cancer Research Centre Institutional Animal Care and Use Committee, Telethon Kids Institute Animal Ethics Committee or The University of Queensland Molecular Biosciences committee. This study involved no human participants. All research with PDX conformed to the principles of the Helsinki Declaration.

\section{Consent for publication}

Not applicable.

\section{Competing interests}

The authors declare that they have no competing interests.

\section{Author details}

${ }^{1}$ The University of Queensland Diamantina Institute, The University of Queensland, Woolloongabba, QLD 4102, Australia. ${ }^{2}$ Institute for Molecular Bioscience, The University of Queensland, St Lucia, QLD 4072, Australia. ${ }^{3}$ ARC Centre of Excellence for Convergent Bio-Nano Science and Technology, The
University of Queensland, St Lucia, QLD 4072, Australia. ${ }^{4}$ Australian Institute for Bioengineering and Nanotechnology, The University of Queensland, St Lucia, QLD 4072, Australia. ${ }^{5}$ Clinical Research Division, Fred Hutchinson Cancer Research Center, Seattle, WA 98109, USA. ${ }^{6}$ Brain Tumour Research Program, Telethon Kids Cancer Centre, Telethon Kids Institute, Nedlands, WA 6009, Australia. ${ }^{7}$ Bioinformatics Division, The Walter and Eliza Hall Institute of Medical Research, Parkville, Victoria 3052, Australia. ${ }^{8}$ Department of Medical Biology, Faculty of Medicine, Dentistry and Health Sciences, The University of Melbourne, Melbourne, Victoria 3010, Australia. ${ }^{9}$ QFAB Bioinformatics, Institute for Molecular Bioscience, The University of Queensland, St Lucia, QLD 4072, Australia. ${ }^{10}$ Programme in Developmental and Stem Cell Biology, Arthur and Sonia Labatt Brain Tumour Research Centre, Hospital for Sick Children, Toronto, Ontario MSG 1X8, Canada. ${ }^{11}$ Division of Neurosurgery, Hospital for Sick Children, Toronto, Ontario MSG 1X8, Canada. ${ }^{12}$ Department of Clinical Pathology, Faculty of Medicine, Dentistry and Health Sciences, The University of Melbourne, Melbourne, Victoria 3010, Australia.

Received: 23 July 2020 Accepted: 4 June 2021

Published online: 21 June 2021

\section{References}

1. Pui CH, Gajjar AJ, Kane JR, Qaddoumi IA, Pappo AS. Challenging issues in pediatric oncology. Nat Rev Clin Oncol. 2011;8(9):540-9. https://doi.org/10.1 038/nrclinonc.2011.95.

2. Thompson MC, Fuller C, Hogg TL, Dalton J, Finkelstein D, Lau CC, et al. Genomics identifies medulloblastoma subgroups that are enriched for specific genetic alterations. J Clin Oncol. 2006;24(12):1924-31. https://doi. org/10.1200/JCO.2005.04.4974.

3. Kool M, Koster J, Bunt J, Hasselt NE, Lakeman A, van Sluis P, et al. Integrated genomics identifies five medulloblastoma subtypes with distinct genetic profiles, pathway signatures and clinicopathological features. PLoS One. 2008;3(8):e3088. https://doi.org/10.1371/journal.pone.0003088.

4. Cho YJ, Tsherniak A, Tamayo P, Santagata S, Ligon A, Greulich H, et al. Integrative genomic analysis of medulloblastoma identifies a molecular subgroup that drives poor clinical outcome. J Clin Oncol. 2011;29(11):142430. https://doi.org/10.1200/JCO.2010.28.5148.

5. Northcott PA, Korshunov A, Witt H, Hielscher T, Eberhart CG, Mack S, et al. Medulloblastoma comprises four distinct molecular variants. J Clin Oncol. 2011:29(11):1408-14. https://doi.org/10.1200/JCO.2009.27.4324.

6. Taylor MD, Northcott PA, Korshunov A, Remke M, Cho YJ, Clifford SC, et al Molecular subgroups of medulloblastoma: the current consensus. Acta Neuropathol. 2012;123(4):465-72. https://doi.org/10.1007/s00401-011-0922-z.

7. Cavalli FMG, Remke M, Rampasek L, Peacock J, Shih DJH, Luu B, et al. Intertumoral heterogeneity within medulloblastoma subgroups. Cancer Cell. 2017;31(6):737-54 e6. https://doi.org/10.1016/j.ccell.2017.05.005.

8. Ellison DW, Onilude OE, Lindsey JC, Lusher ME, Weston CL, Taylor RE, et al. beta-Catenin status predicts a favorable outcome in childhood medulloblastoma: the United Kingdom Children's Cancer Study Group Brain Tumour Committee. J Clin Oncol. 2005;23(31):7951-7. https://doi.org/10.12 00/JCO.2005.01.5479.

9. Rutkowski S, Bode U, Deinlein F, Ottensmeier H, Warmuth-Metz M, Soerensen N, et al. Treatment of early childhood medulloblastoma by postoperative chemotherapy alone. N Engl J Med. 2005;352(10):978-86. https://doi.org/10.1056/NEJMoa042176.

10. Gajjar A, Chintagumpala M, Ashley D, Kellie S, Kun LE, Merchant TE, et al. Risk-adapted craniospinal radiotherapy followed by high-dose chemotherapy and stem-cell rescue in children with newly diagnosed medulloblastoma (St Jude Medulloblastoma-96): long-term results from a prospective, multicentre trial. Lancet Oncol. 2006;7(10):813-20. https://doi. org/10.1016/S1470-2045(06)70867-1.

11. Packer RJ, Gajjar A, Vezina G, Rorke-Adams L, Burger PC, Robertson PL, et al. Phase III study of craniospinal radiation therapy followed by adjuvant chemotherapy for newly diagnosed average-risk medulloblastoma. J Clin Oncol. 2006;24(25):4202-8. https://doi.org/10.1200/JCO.2006.06.4980.

12. Remke M, Ramaswamy V, Taylor MD. Medulloblastoma molecular dissection: the way toward targeted therapy. Curr Opin Oncol. 2013;25(6):674-81. https://doi.org/10.1097/CCO.0000000000000008.

13. Kainthla R, Kim KB, Falchook GS. Dabrafenib for treatment of BRAF-mutant melanoma. Pharmgenomics Pers Med. 2014;7:21-9. https://doi.org/10.2147/ PGPM.S37220. 
14. Verma S, Miles D, Gianni L, Krop IE, Welslau M, Baselga J, et al. Trastuzumab emtansine for HER2-positive advanced breast cancer. N Engl J Med. 2012; 367(19):1783-91. https://doi.org/10.1056/NEJMoa1209124.

15. Rusert JM, Juarez EF, Brabetz S, Jensen J, Garancher A, Chau LQ, et al. Functional precision medicine identifies new therapeutic candidates for medulloblastoma. Cancer Res. 2020;80(23):5393-407. https://doi.org/10.11 58/0008-5472.CAN-20-1655.

16. Genovesi LA, Ng CG, Davis MJ, Remke M, Taylor MD, Adams DJ, et al. Sleeping Beauty mutagenesis in a mouse medulloblastoma model defines networks that discriminate between human molecular subgroups. Proc Natl Acad Sci U S A. 2013;110(46):E4325-34. https://doi. org/10.1073/pnas.1318639110.

17. Jeon J, Nim S, Teyra J, Datti A, Wrana JL, Sidhu SS, et al. A systematic approach to identify novel cancer drug targets using machine learning, inhibitor design and high-throughput screening. Genome Med. 2014;6(7):57. https://doi.org/10.1186/s13073-014-0057-7.

18. Markant SL, Esparza LA, Sun J, Barton KL, McCoig LM, Grant GA, et al. Targeting sonic hedgehog-associated medulloblastoma through inhibition of Aurora and Polo-like kinases. Cancer Res. 2013;73(20):6310-22. https://doi. org/10.1158/0008-5472.CAN-12-4258.

19. Bandopadhayay P, Bergthold G, Nguyen B, Schubert S, Gholamin S, Tang Y, et al. BET bromodomain inhibition of MYC-amplified medulloblastoma. Clin Cancer Res. 2014;20(4):912-25. https://doi.org/10.1158/1078-0432.CCR-13-2281.

20. Cook Sangar ML, Genovesi LA, Nakamoto MW, Davis MJ, Knoblaugh SE, Ji P, et al. Inhibition of CDK4/6 by Palbociclib Significantly Extends Survival in Medulloblastoma Patient-Derived Xenograft Mouse Models. Clin Cancer Res. 2017;23(19):5802-13. https://doi.org/10.1158/1078-0432.CCR-16-2943.

21. Bandopadhayay P, Piccioni F, O'Rourke R, Ho P, Gonzalez EM, Buchan G, et al. Neuronal differentiation and cell-cycle programs mediate response to BET-bromodomain inhibition in MYC-driven medulloblastoma. Nat Commun. 2019;10(1):2400. https://doi.org/10.1038/s41467-019-10307-9.

22. Shannon P, Markiel A, Ozier O, Baliga NS, Wang JT, Ramage D, et al. Cytoscape: a software environment for integrated models of biomolecular interaction networks. Genome Res. 2003;13(11):2498-504. https://doi.org/1 $0.1101 /$ gr.1239303.

23. Wishart DS, Knox C, Guo AC, Shrivastava S, Hassanali M, Stothard P, et al DrugBank: a comprehensive resource for in silico drug discovery and exploration. Nucleic Acids Res. 2006;34(Database issue):D668-72. https://doi. org/10.1093/nar/gkj067.

24. Orchard S, Kerrien S, Abbani S, Aranda B, Bhate J, Bidwell S, et al. Protein interaction data curation: the International Molecular Exchange (IMEx) consortium. Nat Methods. 2012;9(4):345-50. https://doi.org/10.103 8/nmeth.1931.

25. Brabetz S, Leary SES, Grobner SN, Nakamoto MW, Seker-Cin H, Girard EJ, et al. A biobank of patient-derived pediatric brain tumor models. Nat Med. 2018;24(11):1752-61. https://doi.org/10.1038/s41591-018-0207-3.

26. O'Brien J, Hayder H, Peng C. Automated quantification and analysis of cell counting procedures using ImageJ plugins. J Vis Exp. 2016;117:e54719.

27. Duan S, Bleibel WK, Huang RS, Shukla SJ, Wu X, Badner JA, et al. Mapping genes that contribute to daunorubicin-induced cytotoxicity. Cancer Res. 2007;67(11):5425-33. https://doi.org/10.1158/0008-5472.CAN-06-4431.

28. Huang RS, Duan S, Bleibel WK, Kistner EO, Zhang W, Clark TA, et al. A genome-wide approach to identify genetic variants that contribute to etoposide-induced cytotoxicity. Proc Natl Acad Sci U S A. 2007;104(23): 9758-63. https://doi.org/10.1073/pnas.0703736104.

29. Jeong $H$, Mason SP, Barabasi AL, Oltvai ZN. Lethality and centrality in protein networks. Nature. 2001;411(6833):41-2. https://doi.org/10.1038/3 5075138.

30. Li J, Ren J, Sun W. Systematic review of ixabepilone for treating metastatic breast cancer. Breast Cancer. 2017;24(2):171-9. https://doi.org/10.1007/s122 82-016-0717-0.

31. Vilmar A, Garcia-Foncillas J, Huarriz M, Santoni-Rugiu E, Sorensen JB. RT-PCR versus immunohistochemistry for correlation and quantification of ERCC1, BRCA1, TUBB3 and RRM1 in NSCLC. Lung Cancer. 2012;75(3):306-12. https:// doi.org/10.1016/j.lungcan.2011.08.016.

32. Parker AL, Teo WS, McCarroll JA, Kavallaris M. An emerging role for tubulin isotypes in modulating cancer biology and chemotherapy resistance. Int J Mol Sci. 2017;18(7):1434.

33. Moen MD. Ixabepilone: in locally advanced or metastatic breast cancer. Drugs. 2009;69(11):1471-81. https://doi.org/10.2165/00003495-20096911000006.
34. Lopus M, Smiyun G, Miller H, Oroudjev E, Wilson L, Jordan MA. Mechanism of action of ixabepilone and its interactions with the betalll-tubulin isotype. Cancer Chemother Pharmacol. 2015;76(5):1013-24. https://doi.org/10.1007/ s00280-015-2863-z.

35. Yamaguchi H, Paranawithana SR, Lee MW, Huang Z, Bhalla KN, Wang HG. Epothilone B analogue (BMS-247550)-mediated cytotoxicity through induction of Bax conformational change in human breast cancer cells. Cancer Res. 2002;62(2):466-71.

36. Yamaguchi H, Chen J, Bhalla K, Wang HG. Regulation of Bax activation and apoptotic response to microtubule-damaging agents by p53 transcriptiondependent and -independent pathways. J Biol Chem. 2004;279(38):39431-7. https://doi.org/10.1074/jbc.M401530200.

37. Teicher BA. Tumor models for efficacy determination. Mol Cancer Ther. 2006;5(10):2435-43. https://doi.org/10.1158/1535-7163.MCT-06-0391.

38. Huang RS, Duan S, Kistner EO, Bleibel WK, Delaney SM, Fackenthal DL, et al. Genetic variants contributing to daunorubicin-induced cytotoxicity. Cancer Res. 2008;68(9):3161-8. https://doi.org/10.1158/0008-5472.CAN-07-6381.

39. Li L, Fridley B, Kalari K, Jenkins G, Batzler A, Safgren S, et al. Gemcitabine and cytosine arabinoside cytotoxicity: association with lymphoblastoid cell expression. Cancer Res. 2008;68(17):7050-8. https://doi.org/10.1158/0008-54 72.CAN-08-0405.

40. Yu KH, Ricigliano M, Hidalgo M, Abou-Alfa GK, Lowery MA, Saltz LB, et al. Pharmacogenomic modeling of circulating tumor and invasive cells for prediction of chemotherapy response and resistance in pancreatic cancer. Clin Cancer Res. 2014;20(20):5281-9. https://doi.org/10.1158/1078-0432. CCR-14-0531.

41. Northcott PA, Shih DJ, Peacock J, Garzia L, Morrissy AS, Zichner T, et al. Subgroup-specific structural variation across 1,000 medulloblastoma genomes. Nature. 2012;488(7409):49-56. https://doi.org/10.1038/nature11327.

42. Gajjar A, Stewart CF, Ellison DW, Kaste S, Kun LE, Packer RJ, et al. Phase I study of vismodegib in children with recurrent or refractory medulloblastoma: a pediatric brain tumor consortium study. Clin Cancer Res. 2013;19(22):6305-12. https://doi.org/10.1158/1078-0432.CCR-13-1425.

43. Robinson GW, Orr BA, Wu G, Gururangan S, Lin T, Qaddoumi I, et al. Vismodegib exerts targeted efficacy against recurrent sonic hedgehogsubgroup medulloblastoma: results from phase II pediatric brain tumor consortium studies PBTC-025B and PBTC-032. J Clin Oncol. 2015;33(24): 2646-54. https://doi.org/10.1200/JCO.2014.60.1591.

44. Pei Y, Liu KW, Wang J, Garancher A, Tao R, Esparza LA, et al. HDAC and PI3K antagonists cooperate to inhibit growth of MYC-driven medulloblastoma. Cancer Cell. 2016;29(3):311-23. https://doi.org/10.1016/j.ccell.2016.02.011.

45. Pugh TJ, Weeraratne SD, Archer TC, Pomeranz Krummel DA, Auclair D, Bochicchio J, et al. Medulloblastoma exome sequencing uncovers subtypespecific somatic mutations. Nature. 2012;488(7409):106-10. https://doi.org/1 $0.1038 /$ nature 11329

46. Jones DT, Jager N, Kool M, Zichner T, Hutter B, Sultan M, et al. Dissecting the genomic complexity underlying medulloblastoma. Nature. 2012; 488(7409):100-5. https://doi.org/10.1038/nature11284.

47. Tang Y, Gholamin S, Schubert S, Willardson MI, Lee A, Bandopadhayay P, et al. Epigenetic targeting of Hedgehog pathway transcriptional output through BET bromodomain inhibition. Nat Med. 2014;20(7):732-40. https:// doi.org/10.1038/nm.3613.

48. Venkataraman S, Alimova I, Balakrishnan I, Harris P, Birks DK, Griesinger A, et al. Inhibition of BRD4 attenuates tumor cell self-renewal and suppresses stem cell signaling in MYC driven medulloblastoma. Oncotarget. 2014;5(9): 2355-71. https://doi.org/10.18632/oncotarget.1659.

49. Ahmad Z, Jasnos L, Gil V, Howell L, Hallsworth A, Petrie K, et al. Molecular and in vivo characterization of cancer-propagating cells derived from MYCN-dependent medulloblastoma. PLoS One. 2015;10(3):e0119834. https:// doi.org/10.1371/journal.pone.0119834.

50. Hill RM, Kuijper S, Lindsey JC, Petrie K, Schwalbe EC, Barker K, et al. Combined MYC and P53 defects emerge at medulloblastoma relapse and define rapidly progressive, therapeutically targetable disease. Cancer Cell. 2015;27(1):72-84. https://doi.org/10.1016/j.ccell.2014.11.002.

51. Hanaford AR, Archer TC, Price A, Kahlert UD, Maciaczyk J, Nikkhah G, et al. DiSCoVERing innovative therapies for rare tumors: combining genetically accurate disease models with in silico analysis to identify novel therapeutic targets. Clin Cancer Res. 2016;22(15):3903-14. https://doi.org/10.1158/10780432.CCR-15-3011.

52. Galsky MD, Dritselis A, Kirkpatrick P, Oh WK. Cabazitaxel. Nat Rev Drug Discov. 2010;9(9):677-8. https://doi.org/10.1038/nrd3254. 
53. Northcott PA, Robinson GW, Kratz CP, Mabbott DJ, Pomeroy SL, Clifford SC, et al. Medulloblastoma. Nat Rev Dis Primers. 2019;5(1):11. https://doi.org/1 0.1038/s41572-019-0063-6.

54. Boyle FM, Eller SL, Grossman SA. Penetration of intra-arterially administered vincristine in experimental brain tumor. Neuro Oncol. 2004;6(4):300-5. https://doi.org/10.1215/S1152851703000516.

55. Wang F, Zhou F, Kruh GD, Gallo JM. Influence of blood-brain barrier efflux pumps on the distribution of vincristine in brain and brain tumors. Neuro Oncol. 2010;12(10):1043-9. https://doi.org/10.1093/neuonc/noq056.

56. Winter SS, Ricci J, Luo L, Lovato DM, Khawaja HM, Serna-Gallegos T, et al. ATP Binding Cassette C1 (ABCC1/MRP1)-mediated drug efflux contributes to disease progression in T-lineage acute lymphoblastic leukemia. Health (IIvine Calif). 2013;5(5A):41-50.

57. Girard E, Ditzler S, Lee D, Richards A, Yagle K, Park J, et al. Efficacy of cabazitaxel in mouse models of pediatric brain tumors. Neuro Oncol. 2015; 17(1):107-15. https://doi.org/10.1093/neuonc/nou163.

58. Roque DM, Bellone S, Buza N, Romani C, Cocco E, Bignotti E, et al. Class III beta-tubulin overexpression in ovarian clear cell and serous carcinoma as a maker for poor overall survival after platinum/taxane chemotherapy and sensitivity to patupilone. Am J Obstet Gynecol. 2013;209(1):62 e1-9.

59. Roque DM, Bellone S, English DP, Buza N, Cocco E, Gasparrini S, et al. Tubulinbeta-lll overexpression by uterine serous carcinomas is a marker for poor overall survival after platinum/taxane chemotherapy and sensitivity to epothilones. Cancer. 2013;119(14):2582-92. https://doi.org/10.1002/cncr.28017.

60. Oehler C, von Bueren AO, Furmanova P, Broggini-Tenzer A, Orlowski K, Rutkowski $\mathrm{S}$, et al. The microtubule stabilizer patupilone (epothilone B) is a potent radiosensitizer in medulloblastoma cells. Neuro Oncol. 2011;13(9): 1000-10. https://doi.org/10.1093/neuonc/nor069.

61. Taylor, Mark D. Expression data from normal human cerebellum. Expression profiling by array. Gene Expression Omnibus. 2021. https:/www.ncbi.nlm. nih.gov/geo/query/acc.cgi?acc=GSE167447.

\section{Publisher's Note}

Springer Nature remains neutral with regard to jurisdictional claims in published maps and institutional affiliations.

Ready to submit your research? Choose BMC and benefit from:

- fast, convenient online submission

- thorough peer review by experienced researchers in your field

- rapid publication on acceptance

- support for research data, including large and complex data types

- gold Open Access which fosters wider collaboration and increased citations

- maximum visibility for your research: over $100 \mathrm{M}$ website views per year

At $\mathrm{BMC}$, research is always in progress.

Learn more biomedcentral.com/submissions 